\title{
Test-Pattern Selection for Screening Small-Delay Defects in Very-Deep Submicrometer Integrated Circuits
}

\author{
Mahmut Yilmaz, Member, IEEE, Krishnendu Chakrabarty, Fellow, IEEE, and \\ Mohammad Tehranipoor, Senior Member, IEEE
}

\begin{abstract}
Timing-related defects are major contributors to test escapes and in-field reliability problems for very-deep submicrometer integrated circuits. Small delay variations induced by crosstalk, process variations, power-supply noise, as well as resistive opens and shorts can potentially cause timing failures in a design, thereby leading to quality and reliability concerns. We present a test-grading technique that uses the method of output deviations for screening small-delay defects (SDDs). A new gate-delay defect probability measure is defined to model delay variations for nanometer technologies. The proposed technique intelligently selects the best set of patterns for SDD detection from an $n$-detect pattern set generated using timing-unaware automatic test-pattern generation (ATPG). It offers significantly lower computational complexity and excites a larger number of long paths compared to a current generation commercial timing-aware ATPG tool. Our results also show that, for the same pattern count, the selected patterns provide more effective coverage ramp-up than timing-aware ATPG and a recent patternselection method for random SDDs potentially caused by resistive shorts, resistive opens, and process variations.
\end{abstract}

Index Terms-Delay test, output deviations, process variations, small-delay defects, test-pattern grading.

\section{INTRODUCTION}

$\mathbf{V}$ ERY DEEP submicrometer (VDSM) process technologies are leading to increasing densities and higher clock frequencies for integrated circuits (ICs). However, VDSM technologies are especially susceptible to process variations, crosstalk noise, power-supply noise, and defects such as resistive shorts and opens, which induce small delay variations in the circuit components. Such delay variations are referred to as small-delay defects (SDDs) in the literature [1], [2].

Manuscript received October 17, 2008; revised February 10, 2009, September 15, 2009, and December 11, 2009. Current version published April 21, 2010. The work of M. Yilmaz and K. Chakrabarty was supported in part by the Semiconductor Research Corporation (SRC), under contract 1588, and by the National Science Foundation (NSF), under Grant ECCS-0823835. The work of M. Tehranipoor was supported in part by SRC, under contracts 1455 and 1587, and by NSF, under Grant ECCS-0823992. This paper was recommended by Associate Editor, R. D. (Shawn) Blanton.

M. Yilmaz is with the Design-for-Test Team, Advanced Micro Devices, Inc., Sunnyvale, CA 94085 USA (e-mail: mahmut.yilmaz@amd.com).

K. Chakrabarty is with the Department of Electrical and Computer Engineering, Duke University, Durham, NC 27708 USA (e-mail: krish@ee.duke.edu).

M. Tehranipoor is with the Department of Electrical and Computer Engineering, University of Connecticut, Storrs, CT 06269 USA (e-mail: tehrani@engr.uconn.edu).

Color versions of one or more of the figures in this paper are available online at http://ieeexplore.ieee.org.

Digital Object Identifier 10.1109/TCAD.2010.2043591
Although the delay introduced by each SDD is small, the overall impact can be significant if the target path is critical, has low slack, or includes many SDDs. The overall delay of the path may become longer than the clock period, causing circuit failure or temporarily incorrect results. As a result, the detection of SDDs typically requires fault excitation through leastslack paths. The longest paths in the circuit, except false paths and multi-cycle paths, are referred to as the least-slack paths.

The transition delay-fault (TDF) [3] model attempts to propagate the lumped delay defect of a gate by logical transitions to the observation points or state elements. The effectiveness of the TDF model for SDDs has often been questioned [1], [4] due to its tendency to excite transition delay-faults through short paths [1].

Due to the growing interest in SDDs, the first commercial timing-aware automatic test-pattern generation (ATPG) tools were introduced recently, e.g., new versions of Mentor Graphics FastScan, Cadence Encounter Test, and Synopsys TetraMax [5]-[7]. These tools attempt to make ATPG patterns more effective for SDDs by exercising longer paths or applying patterns at higher-than-rated clock frequencies. However, only a limited amount of timing information is supplied to these tools, either via standard delay format (SDF) files (for FastScan and Encounter Test) or through a static timing analysis (STA) tool (for TetraMax). As a result, none of these tools can be easily extended to take into account process variations, crosstalk, power-supply noise, or similar SDD-inducing effects on path delays. These tools simply rely on the assumption that the longest paths (determined using STA or SDF data) in a design are more prone to failure due to SDDs. Moreover, the test generation time increases considerably when these tools are run in timing-aware mode. Fig. 1 shows a comparison of the run times of two current generation ATPG tools from the same EDA company: 1) timing-unaware ATPG, i.e., a traditional transition-delay-fault pattern generator; and 2) timing-aware ATPG that takes timing information into account. The results are shown for some of the International Workshop on Logic and Synthesis (IWLS) 2005 benchmarks [8] and the absolute run times are shown in [9]. It can be seen from Fig. 1 that, when the benchmark is large, the timing-aware ATPG takes significantly more central processing unit (CPU) time, e.g., as much as 209 times more for the "netcard" benchmark. The CPU times in Fig. 1 reflect distributed ATPG results using 


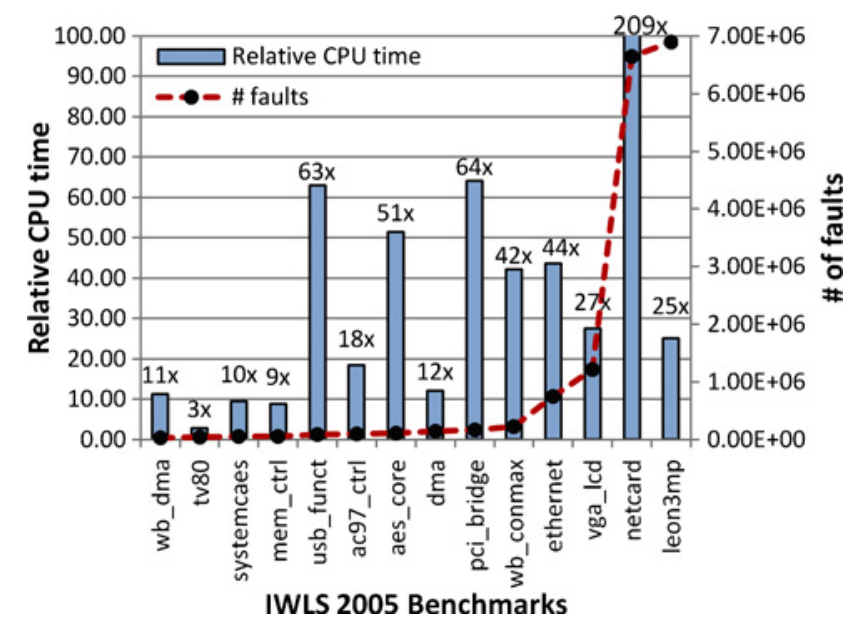

Fig. 1. Comparison of ATPG run times (CPU time): timing-aware ATPG relative to timing-unaware ATPG for IWLS'2005 benchmarks.

eight processors, and the numbers are normalized such that the run-time of timing-unaware ATPG is taken to be one unit.

The complexity of today's ICs and shrinking process technologies are also leading to prohibitively high test-data volumes. For example, the volume for TDFs is two to five times higher than that for stuck-at faults [10], and it has been demonstrated recently that test patterns for such sequence and timing-dependent faults are more important for newer technologies [11]. The 2007 International Technology Roadmap for Semiconductors predicted that the test data volume for integrated circuits will be as much as 38 times larger and the test application time will be about 17 times longer in 2015 than it was in 2007 [12]. Therefore, efficient pattern-selection methods are required to reduce the total pattern count while effectively targeting SDDs.

This paper presents the output deviation measure [13], [14] as a surrogate coverage metric for SDDs and a testpattern grading method to select the best patterns for SDD detection from a large repository test set. A flexible, but general, probabilistic fault model is used to target defects. The proposed method can be used with traditional, timing-unaware ATPG tools to generate a high-quality and compact delay-fault pattern set. It can also be used to select the most effective patterns from large timing-aware test sets. Experimental results show that the proposed method can effectively select the highest quality patterns from large test sets that cannot be used in their entirety for production test environments with tight pattern-count limits. It also considers process-variabilityinduced delay variations, unlike most previous methods. The proposed approach requires significantly less CPU time than a commercial timing-aware ATPG tool under pattern-count limits. For various metrics, namely coverage of long paths, detection of injected defects, and coverage ramp-up, it is shown to outperform a commercial timing-aware ATPG tool. We also compare the proposed method with the approach proposed by Lee et al. [15], in which path-length calculations are approximated for better run-time, and we highlight better long-path coverage, lower run times, and faster coverage rampup for injected faults.
In the remainder of this paper, Section II presents related prior work in the area of SDDs. In Section III, we describe the probabilistic fault model and the output deviations metric. Section III-D presents the proposed pattern-selection procedures. In Section IV, we evaluate the proposed method for benchmark circuits and $n$-detection TDF test sets. We also conduct simulated defect-injection experiments to evaluate the effectiveness of the selected patterns for detecting small delays caused by resistive shorts and opens. Section V concludes this paper.

\section{RELATED PRIOR WORK}

SDDs were first alluded to in [16]. In recent years, highquality delay-fault pattern generation for SDDs has received increasing attention. Most of the work is aimed at finding the longest paths in a circuit. Gupta et al. [17] have proposed the as late as possible transition fault model, which attempts to launch one or more transitions at the fault site as late as possible, i.e., through the least-slack path using robust tests. This method suffers from the need for a complex, time-consuming search procedure and robust test-generation constraints. Qui et al. [18] attempt to find the $k$-longest paths (referred to as KLPG) through the inputs and output of each gate for slow-to-rise and slow-to-fall faults. Similar to [17], a considerable amount of pre-processing is needed to search for long paths. Furthermore, a long path through a gate may be a short path in the circuit; thus, not all the paths determined by the method are least-slack paths. Ahmed et al. [1] use STA tools to find long, intermediate (IP), and short paths (SP) to each observation point. Using a timing-unaware ATPG tool, 15-detect transition test patterns are generated. During pattern generation, constraints are applied on IP and SP observation points to mask them. In this way, the ATPG tool is forced to generate patterns for LPs. In the post-processing phase, a pattern-selection algorithm is used to pick patterns that activate the largest number of end-points. Similar to previous methods, a time-consuming search procedure is needed for determining long paths and for path classification. A functional delay-fault test generation method is proposed in [19]. This method generates sequences of instructions for testing delay faults. However, it requires a fault-free unit that can run the instructions for the test program. Similar to earlier methods, this scheme also involves a lengthy preprocessing step. In [20], delay defects within slack intervals are detected by using a clock frequency higher than the rated clock frequency. This method uses a good neighboring die to test the surrounding dies. The responses of the good die and the other dies are compared with each other to find delay defects. A new fault model, called the "transition path delay fault model," is described in [21]. This fault model relaxes the robustness constraint required by the path-delay fault model, and aims to excite paths that are missed by the path-delay fault model. [22] presents a method to accurately determine the fault coverage of path-delay tests by analyzing path reconvergences. This method is applicable to the bounded gate-delay model.

As a result of increasing industry concern regarding SDDs, companies such as Mentor Graphics, Cadence, and Synopsys have recently released timing-aware ATPG tools [2], [5]-[7]. Lin et al. [2] use SDF files to guide ATPG to generate 
transition test through long paths. In a pre-processing step, the proposed method evaluates the delay for "activation" and "propagation" paths for each gate to find longest paths. Test generation is guided by the results of this pre-processing step. Although approximation methods are used to decrease the overhead associated with delay and path-length calculations, this method still takes considerably more time compared to timing-unaware ATPG tool. Kapur et al. [7] use STA toolgenerated pin slack information to guide timing-aware ATPG. Although the pre-processing step is skipped by pushing the slack data calculation to the STA tool, pattern generation takes considerably more time than timing-unaware ATPG.

Statistical static timing analysis (SSTA) can generate variability-aware delay data. It is demonstrated in recent papers [23], [24] that traditional SSTA does not find sensitized paths based on input vectors using statistical data. Furthermore, a complete SSTA flow takes considerable computation time [25], [26]. However, simplified-SSTA-based approaches can be used for pattern selection, as shown in [27], [28]. In [27], authors propose an SSTA-based test pattern quality metric for the detection of SDDs. The computation of the metric requires multiple dynamic timing analysis runs for each test pattern using randomly sampled delay data from Gaussian pin-to-pin delay distributions. The proposed metric is also used for pattern selection. In [28], the authors focus on timing-hazards and propose a timing-hazard-aware SSTA-based pattern selection technique. A qualitative comparison of both of these SSTAbased techniques to our method can be found in Section IV-D.

The "number of activated long paths" is a useful metric for evaluating the quality of delay-fault pattern quality, but a more computationally tractable method is clearly needed. An alternative evaluation method, referred to as the statistical delay quality model (SDQM), has been proposed by Sato et al. [29]. This pattern-grading metric is based on a delay-defect distribution function, which requires delay-defect statistics for fabricated ICs. The method assigns a statistical delay quality level to each test set to evaluate its quality. A drawback of this metric is the need for delay-defect distributions for real chips. This data is not available before volume production and it is difficult and very expensive to obtain it during production test. Another shortcoming of SDQM and similar metrics is that they require knowledge of the longest sensitizable paths, which is not accurately known before production test.

\section{PRoBABIlistic Delay-FAult Model AND OUTPUT DEVIATIONS FOR SDDS}

In this section, we first introduce the concept of gatedelay defect probabilities (DDPs) (Section III-A) and signaltransition probabilities (Section III-B). These probabilities extend the notion of confidence levels, defined in [13] for a single pattern, to pattern-pairs. Next, we show how to use these probability values to propagate the effects of a test pattern to the test observation points (scan flip-flops/primary outputs) (Section III-B). We describe the algorithm used for signalprobability propagation (Section III-C). Finally, we describe how test patterns can be ranked and selected from a large repository (Section III-D).
TABLE I

EXAMPLE DDPM FOR A 2-INPUT OR GATE

\begin{tabular}{|c|c|c|c|c|c|}
\hline & & \multicolumn{4}{|c|}{ Initial Input State } \\
\hline OR & prob & 00 & 01 & 10 & 11 \\
\hline \multirow{2}{*}{ Inputs } & IN0 & 0.5 & 0 & 0.5 & \multirow{2}{*}{0.1} \\
\cline { 2 - 6 } & IN1 & 0.2 & 0.2 & 0 & \\
\hline
\end{tabular}

\section{A. Gate-Delay Defect Probabilities}

DDPs are assigned to each gate in a design. DDPs for a gate are provided in the form of a matrix called the delay defect probability matrix (DDPM). The DDPM for a 2-input OR gate is shown in Table I. The rows in the matrix correspond to each input port of the gate and the columns correspond to the initial input state during a transition.

Assume that the inputs are shown in the order of IN0, IN1. If there is an input transition from " 10 " to " 00 ," the corresponding DDPM column is "10." Since the transition is caused by IN0, the corresponding DDPM row is INO. As a result, the delay-defect probability (DDP) value corresponding to this event is 0.5 . 0.5 , showing the probability that corresponding output transition is delayed beyond a threshold.

For initial state "11," both inputs should switch simultaneously to have an output transition. Corresponding DDPM entries are merged due to this requirement. The entries in Table I have been chosen arbitrarily for the sake of illustration. The real DDPM entries are much smaller than the ones shown in this example.

For an $N$-input gate, the DDPM consists of $N \cdot 2^{N}$ entries, each holding one probability value. If the gate has more than one output, each output of the gate has a different DDPM. Note that the DDP is 0 if the corresponding event cannot provide an output transition. Consider $\operatorname{DDPM}(2,3)$ in Table I. When the initial input state is "10," no change in IN1 can cause an output transition, because the OR gate output is already at high state, and even if IN1 switches to high (1), this will not cause an output transition.

We next discuss how a DDPM is generated. Each entry in DDPM indicates the probability that the delay of a gate is more than a predetermined value, i.e., the critical delay value $\left(T_{\mathrm{CRT}}\right)$. Given the probability density function (PDF) of a delay distribution, the DDP is calculated as

$$
D D P=\operatorname{Prob}\left(x>T_{\mathrm{CRT}}\right)=\int_{T_{\mathrm{CRT}}}^{\infty} \operatorname{PDF}(x) d x .
$$

For instance, if we assume a Gaussian delay distribution for all gates (with mean $\mu$ ) and set the critical delay value to $\mu+X$, each DDP entry can be calculated by replacing $T_{\mathrm{CRT}}$ with $\mu+X$ and using a Gaussian PDF. Note that the delay for each input-to-output transition may have a different mean $(\mu)$ and standard deviation $(\sigma)$. The selection of $X$ is described in Section IV-A.

The delay distribution can be obtained in different ways: 1) using the delay information provided by an SSTA-generated SDF file; 2) using slow, nominal, and fast process corner transistor models; and 3) simulating process variations. In the third method, which is employed in this paper, transistor 
parameters affecting the process variation and the limits of the process variation $(3 \sigma)$ are first determined. Monte Carlo (MC) simulations are next run for each library gate under different capacitive loading and input slew rate conditions. Once the distributions are found for the library gates, depending on the layout, the delay distributions for each individual gate can be updated. Once the distributions are obtained, $T_{\mathrm{CRT}}$ can be appropriately set to compute the DDPM entries. The effects of crosstalk can be simulated separately and the delay distributions of individual gates/wires can be updated accordingly.

The generation of the DDPMs is not the main focus of this paper. We consider DDPMs to be analogous to timing libraries. Our goal is not to develop the most effective techniques for constructing DDPMs; rather, we are using such statistical data to compute deviations and use them for pattern grading and pattern selection. In a standard industrial flow, statistical timing data can be developed by specialized timing groups, so the generation of DDPMs is a pre-processing step and an input to the ATPG-focused test-automation flow.

We have also seen that small changes in the DDPM entries have negligible impact on the pattern-selection results. We attribute this finding to the fact that any DDPM changes affect multiple paths in the circuits, so their impact is amortized over the circuit and the test set. The absolute values of the output deviations are less important than the relative values for different test patterns. Detailed results are presented in Section IV-G and in [9].

\section{B. Propagation of Signal-Transition Probabilities}

Since pattern pairs are required to detect TDFs, there can be a transition on each net of the circuit for every pattern pair. If we assume that there are only two possible logic values for a net, i.e., LOW (L) and HIGH (H), the possible signaltransitions are $L \rightarrow L, L \rightarrow H, H \rightarrow L$, and $H \rightarrow H$. Each of these transitions has a corresponding probability, denoted by $P_{L \rightarrow L}, P_{L \rightarrow H}, P_{H \rightarrow L}$, and $P_{H \rightarrow H}$, respectively, in a vector form $(<\ldots>)$ : $<P_{L \rightarrow L}, P_{L \rightarrow H}, P_{H \rightarrow L}, P_{H \rightarrow H}>$. We refer to this vector as the signal-transition probability (STP) vector. Note that $L \rightarrow L$ or $H \rightarrow H$ implies that the net keeps its value, i.e., no transition occurs.

The nets that are directly connected to the test-application points are called initialization nets (INs). These nets have one of the STPs, corresponding to the applied transition test pattern, equal to 1 . All the other STPs for INs are set to 0 . When signals are propagated through several levels of gates, the STPs can be computed using the DDPM of the gates. Note that interconnects can also have DDPMs to account for crosstalk. In this paper, due to the lack of layout information, we only focus on variations' impact on gate delay. The overall deviation-based framework is, however, general and it can easily accommodate interconnect delay variations if layout information is available, as has been reported in [30].

Definition 1: Let $P_{E}$ be the probability that a net has the expected signal-transition. The deviation on that net is defined by $\Delta=1-P_{E}$. The following rules are applied during the propagation of STPs.
1) If there is no output-signal-transition (output keeps its logic value), then the deviation on the output net is 0 .

2) If there are multiple inputs that can cause the expected signal-transition at the output of a gate, only the inputto-output path that causes the highest deviation at the output net is considered. The other inputs are treated as if they have no effect on the deviation calculation (i.e., they are held at the non-controlling value).

3) When multiple inputs are required to change at the same time to provide the expected output transition, all required input-to-output paths of the gate are considered. Only the unnecessary (redundant) paths are discarded.

A key premise of this paper is that output deviations can be used to compare path lengths. As in the case of path delays, the net deviations also increase as the signal propagates through a sensitized path, a property that follows from the rules used to calculate STPs for a gate output. This claim is formally proven next.

Lemma 1: For any net, let the STP vector be given by $<P_{L \rightarrow L}, P_{L \rightarrow H}, P_{H \rightarrow L}, P_{H \rightarrow H}>$. Among these four probabilities, i.e., $<P_{L \rightarrow L}, P_{L \rightarrow H}, P_{H \rightarrow L}, P_{H \rightarrow H}>$, at least one is non-zero and at most two can be non-zero.

Proof: If there is no signal-value change (the event $L \rightarrow$ $L$ or $H \rightarrow H$ ), the expected STP is 1 and all other probabilities are 0 . If there is a signal-value change, only the expected signal-transition events and the delay-fault case have non-zero probabilities associated with them. The delay-fault case for an expected signal value change of $L \rightarrow H$ is $L \rightarrow L$ (the signal value does not change because of a delay-fault). Similarly, the delay-fault case for an expected signal value change of $H \rightarrow L$ is $H \rightarrow H$.

Theorem 1: The deviation on a net always increases or stays constant on a sensitized path if the signal-probability propagation rules are applied.

Proof: Consider a gate with $K$ inputs and one output. The signal-transition on the output net depends on one of the following cases. From Lemma 1, we note that only two cases need to be considered.

1) Only one of the input-port signal-transitions is enough to create the output signal-transition.

2) Multiple input-port signal-transitions are required to create the output signal-transition.

Let $P_{\text {OUT, }, j}$ be the probability that the gate output makes the expected signal-transition for a given pair of patterns on input $j$, where $1 \leq j \leq K$. Let $\Delta_{\mathrm{OUT}, j}=1-P_{\mathrm{OUT}, j}$ be the deviation for the net corresponding to the gate output.

1) Case (i): Consider a signal-transition on input $j$. Let $Q_{j}$ be the probability of occurrence of this transition. Let $d_{j}$ be the entry in the gate's DDPM that corresponds to the given signal-transition on $j$. The probability that the output makes a signal-transition is given by

$$
P_{\mathrm{OUT}, j}=Q_{j}\left(1-d_{j}\right) .
$$

We assume here that an error at a gate input is independent from the error introduced by the gate. Note that $P_{\mathrm{OUT}, j} \leq Q_{j}$ since $0 \leq d_{j} \leq 1$. Therefore, the probability of getting 


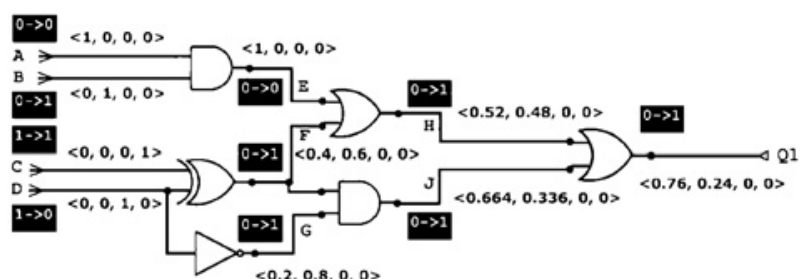

Fig. 2. Example to illustrate the propagation of STPs through the gates of a logic circuit.

TABLE II

EXAMPLE DDPM FOR AND, XOR, INV

\begin{tabular}{|c|c|c|c|c|c|}
\hline & & \multicolumn{4}{|c|}{ Initial Input State } \\
\hline AND & prob & 00 & 01 & 10 & 11 \\
\hline \multirow{2}{*}{ Inputs } & IN0 & \multirow{2}{*}{0.2} & 0.3 & 0 & 0.2 \\
\cline { 2 - 6 } & \multirow{2}{*}{ IN1 } & & 0 & 0.2 & 0.3 \\
\hline \hline \multirow{2}{*}{ XOR } & prob & 00 & 01 & 10 & 11 \\
\hline \multirow{2}{*}{ Inputs } & IN0 & 0.3 & 0.4 & 0.1 & 0.2 \\
\cline { 2 - 6 } & IN1 & 0.3 & 0.4 & 0.2 & 0.4 \\
\hline \hline INV & prob & 0 & 1 & & \\
\hline Inpus & IN0 & 0.2 & 0.2 & & \\
\hline
\end{tabular}

the expected signal-transition decreases and the deviation $\Delta_{\text {OUT, } j}=1-P_{\text {OUT, } j}$ increases (or does not change) as we pass through a gate on a sensitized path. The overall output deviation $\Delta_{\text {OUT }}^{*}$ on the output net is calculated as

$$
\Delta_{\text {OUT }}^{*}=\max _{i \leq j \leq K}\left\{\Delta_{\mathrm{OUT}, j}\right\} .
$$

2) Case (ii): Suppose $L$ input ports $(L>1)$, indexed 1, $2, \ldots, L$, are required to make a transition for the gate output to change. Let $d_{\max }^{*}=\max _{1 \leq j \leq L}\left\{d_{j}\right\}$. The output deviation for the gate in this case is defined as

$$
\Delta_{\mathrm{OUT}}^{*}=\prod_{i=1}^{L} P_{\mathrm{OUT}, i} \cdot\left(1-d_{\max }^{*}\right) \text {. }
$$

Note that $\Delta_{\mathrm{OUT}}^{*} \leq P_{\mathrm{OUT}, i}, 1 \leq i \leq L$, since $0 \leq d_{\mathrm{max}}^{*} \leq 1$. Therefore, we conclude that the probability of getting the expected transition on a net either decreases or remains the same as we pass through a logic gate. In other words, the deviation is monotonically non-decreasing along a sensitized path.

Example: Fig. 2 shows STPs and their propagation for a simple circuit. The test stimuli and the expected fault-free transitions on each net are shown in dark boxes. The calculated STPs are shown in angled brackets $(\langle\ldots\rangle)$. The DDPMs of the gates (OR, AND, XOR, and INV) used in this circuit are given in Tables I and II. The entries in both tables are chosen arbitrarily.

In the following example, the deviations are calculated based on the rules mentioned above for the example circuit in Fig. 2.

1) Net $E$ : There is no output change, which implies that $E$ has the STP $\langle 1,0,0,0\rangle$.
2) Net F: The output changes due to IN1 (net D) of XOR. There is a DDP of 0.4. It implies that with a probability of 0.4 , the output will stay at LOW value, i.e., the STP for net $\mathrm{F}$ is $\langle 0.4,0.6,0,0\rangle$.

3) Net G: Output changes due to INO (net D) of INV, i.e., the STP for net $\mathrm{G}$ is $\langle 0.2,0.8,0,0\rangle$.

4) Net H: Output changes due to IN1 (net F) of OR.

a) If IN1 stays at LOW, output does not change. Therefore, the STP for net $\mathrm{H}$ is $0.4 \odot\langle 1,0,0,0\rangle$, where $\odot$ denotes the dot product.

b) If IN1 goes to HIGH, output changes with a DDP of 0.2 , i.e., the STP for net $\mathrm{H}$ is $0.6 \odot$ $\langle 0.2,0.8,0,0\rangle$.

c) Combining all the above cases, the STP for net $\mathrm{H}$ is $\langle 0.52,0.48 .0,0\rangle$.

5) Net J: Output changes due to both INO (net F) and IN1 (net G) of AND (both required).

a) If both stay at LOW, output does not change, which implies that $\mathrm{J}$ has the STP $0.4 \odot 0.2\langle 1,0,0,0\rangle$.

b) If one of them stays at LOW, output does not change, i.e., the STP for net $\mathrm{J}$ is $0.4 \odot$ $0.8\langle 1,0,0,0\rangle+0.6 \odot 0.2\langle 1,0,0,0\rangle$.

c) If both go to HIGH, the output changes with a DDP. Since both inputs change, we use the maximum DDP, i.e., the STP for net $\mathrm{J}$ is $0.6 \odot$ $0.8 \odot\langle 0.3,0.7,0,0\rangle$.

d) Combining all the above cases, the STP for net J is $\langle 0.664,0.336,0,0\rangle$.

6) Net Q1: The output changes due to only one of the inputs of OR. We need to calculate the deviation for both cases and select the one that causes maximum deviation at the output (Q1).

a) For INO (net $\mathrm{H}$ ) of OR.

i) If IN0 stays at LOW, the output does not change, i.e., the STP for net Q1 is $0.52 \odot$ $\langle 1,0,0,0\rangle$.

ii) If INO goes to $\mathrm{HIGH}$, the output changes with a DDP, i.e., the STP for net Q1 is $0.48 \odot\langle 0.5,0.5,0,0\rangle$.

iii) Combining all the above cases, the STP for net $\mathrm{Q} 1$ is $\langle 0.76,0.24,0,0\rangle$.

b) For IN1 (net J) of OR.

i) If IN1 stays at LOW, the output does not change, i.e., the STP for net Q1 is $0.664 \odot$ $\langle 1,0,0,0\rangle$.

ii) If IN1 goes to HIGH, the output changes with a DDP, i.e., the STP for net Q1 is $0.336 \odot$ $\langle 0.2,0.8,0,0\rangle$.

iii) Combining all the above cases, the STP for net $\mathrm{Q} 1$ is $\langle 0.7312,0.2688,0,0\rangle$.

c) Since INO provided the higher deviation, we finally conclude that the STP for net Q1 is $\langle 0.76,0.24,0,0\rangle$.

Hence, the deviation on Q1 is 0.76 . 


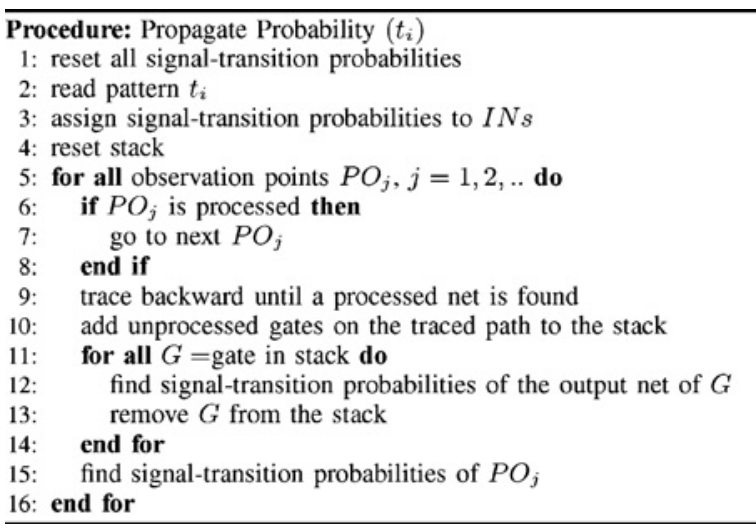

Fig. 3. Signal-transition probability propagation algorithm for calculating output deviations.

\section{Implementation of Algorithm for Propagating Signal- Transition Probabilities}

A depth-first procedure is used to compute STPs for large circuits. When we use a depth-first algorithm, only the nets that are required to find the output deviation on a specific observation point are processed. In this way, a smaller number of gate pointer stacking is required compared to the alternative of simulating the deviations starting from INs and tracing forward.

We first assign STPs to all INs. Then, we start from the observation points (outputs) and backtrace until we find a processed net $(\mathrm{PN})$. A PN has all the signal-transition probabilities assigned. The pseudocode for the algorithm is given in Fig. 3.

If the number of test patterns is $N_{s}$ and the number of nets in the circuit is $N_{n}$, the worst-case time-complexity of the algorithm is $O\left(N_{s} \cdot N_{n}\right)$. However, since the calculation for each pattern is independent of other patterns (we assume fullscan designs in this paper), the algorithm can easily be made multi-threaded. In this case, if the number of threads is $T$, the complexity of the algorithm is reduced to $O\left(\frac{N_{s} \cdot N_{n}}{T}\right)$.

\section{Pattern-Selection Method}

In this subsection, we describe how to use output deviations to select high-quality patterns from an $n$-detect transition-fault pattern set. The number of test patterns to be selected is a user input, e.g., $S$. The parameter $S$ can be set to the number of 1-detect timing-unaware patterns, the number of timing-aware patterns, or any other value that fits the user's test budget.

In our pattern-selection method, we target topological coverage as well as long-path coverage. As a result, we attempt to select patterns that sensitize a wide range of distinct long paths. In this process, we also discard low quality patterns to find a small set of high quality patterns.

For each test observation point $\mathrm{PO}_{j}$, we keep a list of $N_{p}$ most effective patterns in $E F F_{j}$ (Fig. 4, lines 1-3). The patterns in $E F F_{j}$ are the best unique-pattern candidates for exciting a long path through $P O_{j}$. During deviation computation, no pattern $\left(t_{i}\right)$ is added to $E F F_{j}$ if the output deviation at $P O_{j}$ is smaller than a limit ratio ( $D_{\text {LIMIT }}$ ) of the maximum instantaneous output deviation (Fig. 4, line 10). ( $D_{\text {LIMIT }}$ ) can be used to discard low quality patterns. If the output deviation

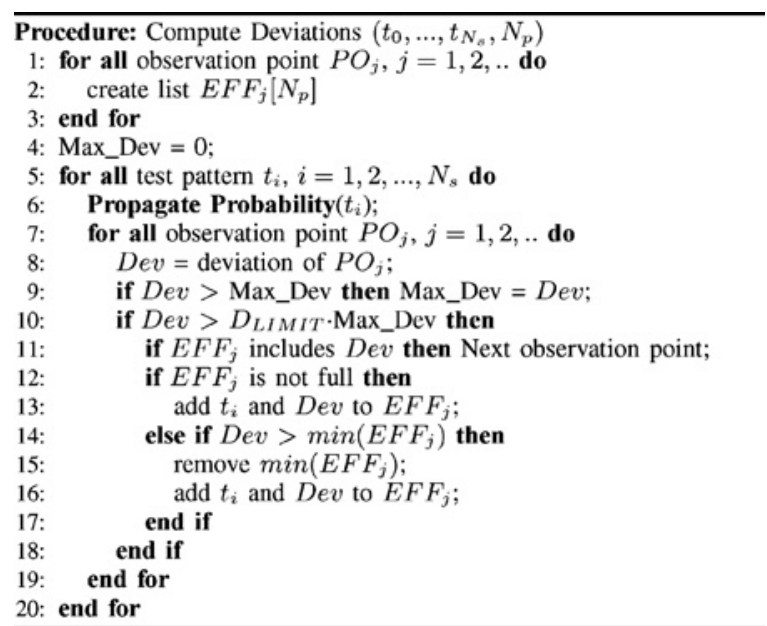

Fig. 4. Deviation-computation algorithm for pattern selection.

is larger than this limit, we first check whether we have added a pattern to $E F F_{j}$ with the same deviation (Fig. 4, line 11). It is unlikely that two different patterns will create the same output deviation on the same output $P O_{j}$ while exciting different non-redundant paths. Since we want a higher topological path-coverage, we skip these cases (Fig. 4, line 11). Although this assumption may not necessarily be true, we assume for the sake of completeness that it holds for most cases. If we observed a unique deviation on $P O_{j}$, we first check whether $E F F_{j}$ is full (already includes $N_{p}$ patterns); see Fig. 4, line 12. Pattern $t_{i}$ is added to $E F F_{j}$ along with its deviation if $E F F_{j}$ is not full or if $t_{i}$ has a larger deviation than the minimum deviation stored in $E F F_{j}$ (Fig. 4, lines 12-17). The effectiveness of a pattern is measured by the number of occurrences of this pattern in $E F F_{j}$ for all values of $j$. For instance, if at the end of deviation computation, pattern $A$ was included in the $E F F$ list for ten observation points, and pattern $B$ was listed in the $E F F$ list for eight observation points, we conclude that pattern $A$ is more effective than pattern $B$.

Once the deviation computation is completed, the list of pattern effectiveness is generated and the final pattern filtering and selection is carried out (Fig. 5). First, pattern effectiveness is generated (Fig. 5, lines 1-9). Since Max_Dev is updated on the fly, we may miss some low quality patterns. As a result, we need to filter by Max_Dev ( $D_{\text {LIMIT }}$ ) again to discard low quality patterns from the final pattern list (Fig. 5, line 5). Setting $D_{\text {LIMIT }}$ to a high value may result in discarding most of the patterns, leaving only the best few patterns. Depending on $D_{\text {LIMIT }}$, the number of remaining patterns can be less than $S$. In the next stage, the patterns are re-sorted by their effectiveness (Fig. 5, line 10). Finally, until the selected pattern number reaches $S$ or all patterns are selected, the top patterns are selected (Fig. 5, line 11). The computational complexity of the selection algorithm is $O\left(N_{s} p\right)$, where $N_{s}$ is the number of test patterns and $p$ is the number of observation points. This procedure is very fast since it only includes two nested for loops and a simple list-item existence check.

\section{E. Alternative Approach}

The two most significant inputs required by the proposed output deviations method are the gate and interconnect delay 


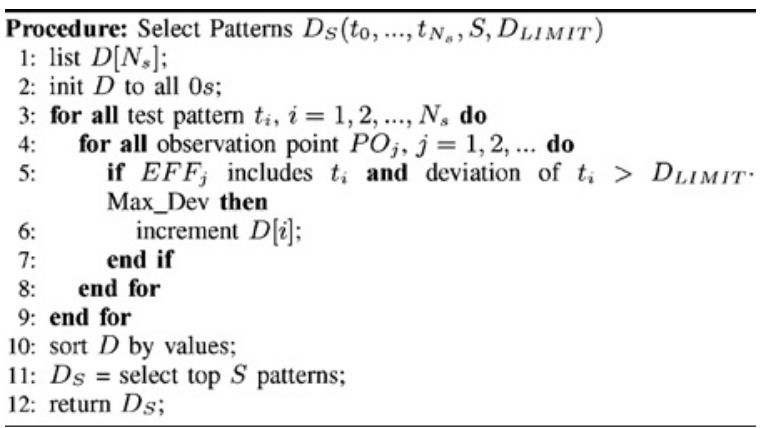

Fig. 5. Pattern selection algorithm.

variations, and $T_{\mathrm{CRT}}$ for gates. Defining a $T_{\mathrm{CRT}}$ for individual gates may not be feasible for all projects. The proposed work is more directly applicable to microprocessor designs with shallow logic depth. For designs with a large number of gates, e.g., more than 15 , on a path, the output deviation metric saturates and equal output deviations (close to 1) are obtained for both long and intermediate paths. Both of these drawbacks can be overcome with slight modifications to the proposed approach as shown in [31]. Deviation-driven pattern selection can also propagate Gaussian distributions instead of defect probabilities. This adds no extra cost since propagation of Gaussians is simply the addition of means and variance (from the central limit theorem of statistics) [31]. In this approach, the dependence of gate level $T_{\mathrm{CRT}}$ is eliminated. $T_{\mathrm{CRT}}$ can be defined for the circuit as a fraction of the functional clock period of the circuit. For each case, the output deviation is defined as the probability that the calculated delay on an observation point (scan flip-flops or primary outputs) is larger than $T_{\mathrm{CRT}}$.

\section{EXPERIMENTAL RESULTS}

In this section, we present experimental results obtained for the IWLS benchmark circuits. We first describe how we obtained DDPs for the logic gates in these benchmarks (Section IV-A). Next, we provide details for the benchmark circuits. Then, we provide details for the experimental setup (Section IV-C). Before presenting the simulation results (Sections IV-F and IV-G), we provide a summary of the dynamictiming simulation method proposed in [15], which will be compared with our proposed deviation-based method (Section IV-E).

\section{A. Finding Gate-Delay Defect Probabilities}

To determine the gate DDPs, we ran 200 HSpice MC simulations on each gate, for all possible input signal-transitions, using $45 \mathrm{~nm}$ process-technology BSIM4 predictive transistor models. We verified that $200 \mathrm{MC}$ simulations are sufficient for generating DDPMs. We observed very little change in the DDPM entries well before we reach 200 MC simulations. Therefore, we conclude that running more $\mathrm{MC}$ simulations will not lead to any significant difference in the DDPM entries.

For each gate type, we simulated the schematic under various loading capacitance and input slew rate conditions to account for spatial correlations. Next, we used interpolation to find the mean and standard deviation of gate delays for individual gate instances. We have seen that interpolation is possible and it is accurate. For instance, the mean delay value approximately linearly changes with the load capacitance and further accuracy can be achieved by using third-order polynomial curve fitting.

MC simulations were carried out using the following realistic process-variation parameters (obtained from a current VDSM technology from industry) for a Gaussian distribution. Transistor gate length $L: 3 \sigma=10 \%$, threshold voltage $V_{T H}: 3 \sigma=30 \%$, and gate-oxide thickness $t_{O X}: 3 \sigma=3 \%$. For each configuration, MC simulations were performed for each possible input transition. For each gate, the probability that the transition-delay value is greater than $T_{\mathrm{CRT}}=N O M+X s$ constitutes the DDP value for the respective input transition (where NOM refers to the nominal delay value). The parameter $X$ is selected in such a way that all the gate instances have at least one non-zero DDPM entry. Note that $X$ is the minimum of all MAX delays (among all gates) for each benchmark. This corresponds to the maximum delay of an inverter driving a single-inverter load. Note that selecting too large a value for $X$ may cause many DDPMs to have all-zero entries, simply because the gate would never have a delay larger than $N O M+X$. As an alternative, $X$ can be set to the $M A X$ delay specified by an STA tool that does not consider process variation effects.

\section{B. Benchmarks}

In this section, we present the details of the IWLS'2005 benchmark circuits. We do not consider the ISCAS benchmarks because these circuits are small and it is easier for an ATPG tool to excite all long paths with a small number of patterns.

IWLS benchmark circuit statistics are shown in Table III. As seen, IWLS benchmarks represent a wide range of application areas, including memory controllers and microprocessors. Note that IWLS benchmarks are provided in the Verilog RTL format, and the statistics given in Table III may slightly change depending on the synthesis tool and synthesis optimization options. For our experiments, we selected a subset of the IWLS benchmarks: systemcaes, usb_funct, ac97_ctrl, aes_core, pci_bridge 32, wb_conmax, and ethernet. The largest benchmarks require a prohibitive amount of computing resources for the collection of simulation data for pattern quality evaluation (sensitized paths and coverage ramp-up), therefore we do not report results for them.

\section{Experimental Setup}

All experiments were performed on a pool of state-ofthe-art servers with at least eight processors available at all times, 16 GB of memory, and running Linux. The program to compute output deviations was implemented using $\mathrm{C}++$. A commercial tool was used to perform Verilog netlist synthesis and scan insertion for the IWLS benchmark circuits. We used a commercial ATPG tool to generate $n$-detect TDF test patterns and timing-aware TDF patterns for these circuits. The ATPG 
TABLE III

BENCHMARK STATISTICS

\begin{tabular}{|l|r|r|r|l|}
\hline \multicolumn{1}{|c|}{ Benchmark } & \# I/O & \# Gates & \# Flip-Flops & Function \\
\hline wb_dma & 942 & 7619 & 563 & WISHBONE DMA/Bridge IP Core \\
\hline tv80 & 103 & 13326 & 359 & TV80 8-Bit Microprocessor Core \\
\hline systemcaes & 387 & 17817 & 670 & SystemC AES \\
\hline mem_ctrl & 208 & 22015 & 1083 & WISHBONE Memory Controller \\
\hline usb_funct & 167 & 25531 & 1746 & USB function core \\
\hline ac97_ctrl & 115 & 28083 & 2199 & WISHBONE AC 97 Controller \\
\hline aes_core & 267 & 29186 & 530 & AES Cipher \\
\hline dma & 120 & 41026 & 2189 & Direct Memory Access (DMA) Controller \\
\hline pci_bridge32 & 361 & 43907 & 3359 & PCI Interface \\
\hline wb_conmax & 388 & 59484 & 770 & WISHBONE Conmax IP Core \\
\hline ethernet & 45 & 153948 & 10544 & Ethernet IP core \\
\hline vga_lcd & 222 & 252302 & 17079 & WISHBONE rev.B2 compliant Enhanced VGA/LCD Controller \\
\hline netcard & 186 & 1356533 & 97831 & Network Card Controller \\
\hline leon3mp & 2546 & 1452517 & 108839 & 32-bit processor compliant with SPARC V8 architecture \\
\hline
\end{tabular}

tool was forced to generate launch-on-capture transition fault patterns. The primary input change during capture cycles and the observation of primary outputs was prevented to simulate realistic test environments. The path delays were calculated using an in-house dynamic path-timing simulator. All simulations were run in parallel on eight processors.

\section{Comparison to SSTA-Based Techniques}

In this section, we qualitatively compare the proposed work to SSTA-based pattern selection methods proposed in [27] and [28]. The summary of this comparison is illustrated in Table IV. Both [27] and the proposed work presents a transition-test pattern quality metric for the detection of SDDs in the presence of process variations. The main focus of [28], on the other hand, is to present a timing-hazard-aware SSTA based technique for the same target defect group. Timinghazards are not covered by [27] or the proposed work. The formulation is different in these methods. In [27], dynamic timing analysis is run multiple times, for each test pattern, to create a delay distribution. Simple operators, e.g., $+/-$, are used while propagating the delay values. In [28], statistical dynamic timing analysis is run once for each test pattern. Similar to [27], simple operators are used for delay propagation, but the analysis of timing hazards adds complexity to the formulation. Both of these methods assume a Gaussian delay distribution. On the other hand, in the proposed work there is no assumption regarding the shape of delay distribution. This is because we use probability values instead of distributions. The metric is computed using probability propagation. The drawback of the proposed method is that its effectiveness drops if the combinational depth of the circuit is very large, i.e., greater than ten. In contrast, both [27] and [28] can handle large combinational depths, and using central limit theorem (CLT), it can even be argued that their accuracy may increase with the increased combinational depth. The run-time of SSTA based approaches [27] and [28] is expected to be a limiting factor in the applicability to industrial size designs. Further optimization may eliminate this shortcoming. On the other hand, the proposed method is quick and its run-time increases less rapidly with increase in circuit size.

\section{E. Dynamic Timing Simulation}

In this paper, we compare our proposed method with the one presented in [15]. Two methods for estimating the path delays for a given test vector are proposed in [15]. In particular, a path-based and a cone-based delay estimation scheme were provided. The estimated delays are called "metrics" associated with the test vectors. Two different delay models are used during the estimation process: 1) unit delay model, each gate has a unit delay, no spatial correlations are considered; and 2) differential delay model, the gate type and the number of fanouts are considered.

For the path-based scheme, it is assumed that a set of critical paths is given. Non-robustly excited critical paths and their corresponding delays are found using either the unit or the differential delay model. The largest delay caused by the test pattern is assigned as the "metric" for the pattern. If there is no non-robust transition on a gate, zero delay is assumed on that gate. Because of the non-robust restriction, delay accuracy is lost. For the cone-based method, there are no non-robust restrictions, but delays are calculated for small cones of logic, and then the delays of cones are added to approximate the overall delay. Once all test vectors are associated with a "metric," the patterns are ordered by the "metric" and the top $1 / 3$ of the patterns are selected.

To compare our work to the dynamic-timing simulation based method, we implemented the cone based scheme using the differential delay model. Instead of the number of fanouts, we used capacitive loading of fanout gates to update the delay associated with each gate instance. The capacitive loading of gate ports is found by running HSpice simulations on transistor-level gate models. Note that in the absence of layouts, this data does not include layout-extracted data such as resistances and capacitances. Once the "metrics" are computed, we selected the top $1 / 3$ of the patterns as proposed by Lee et al. [15]. 
TABLE IV

COMPARISON TO SSTA-BASED APPROACHES

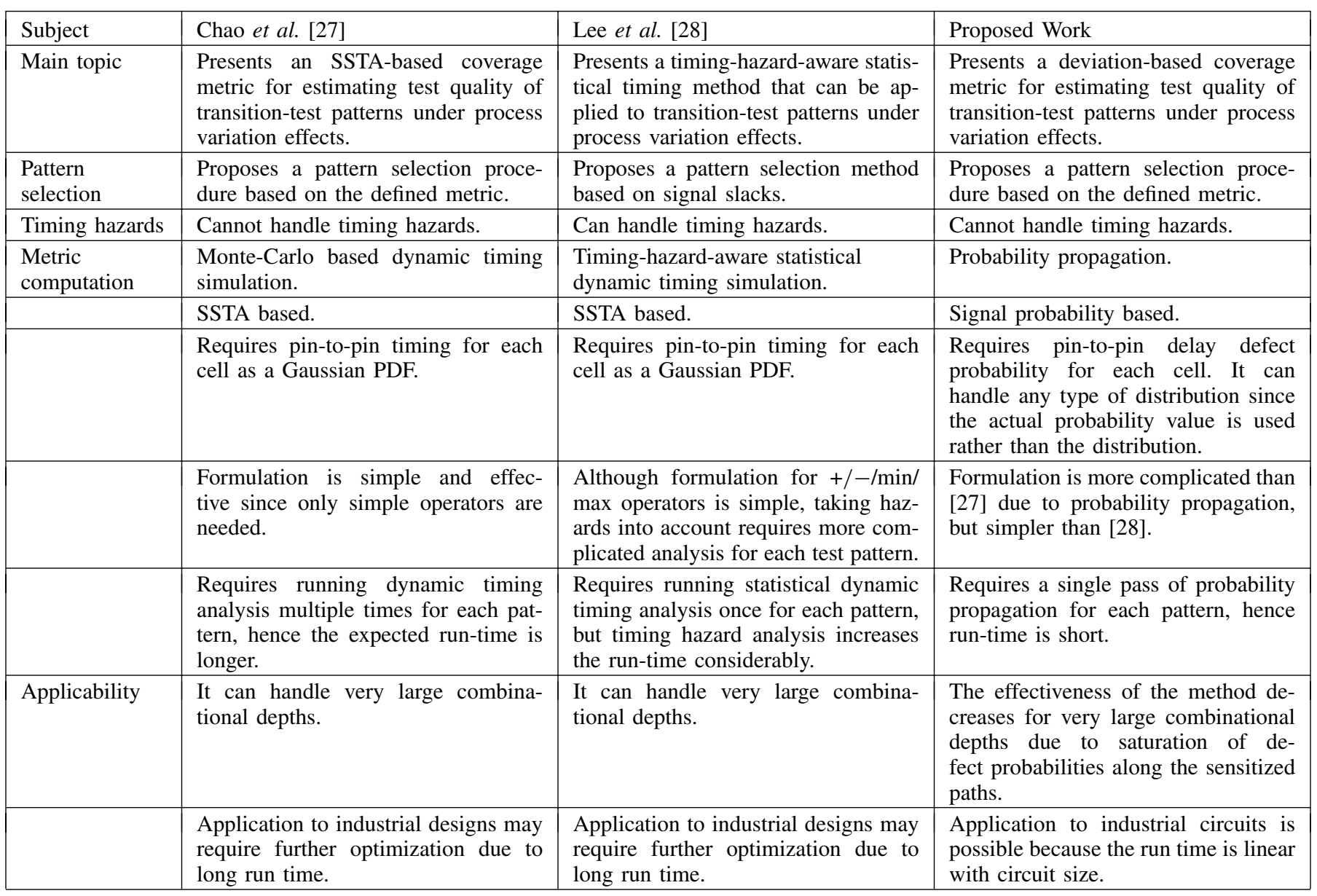

\section{F. Correlation Between Output Deviations and Path Lengths}

We ran correlation analysis to determine the relationship between output deviations and sensitized path lengths. For each benchmark, we calculated output deviations and path lengths for $n$-detect transition-fault test-patterns $(n=1,3,5,8,10)$. We simulated these patterns using the in-house dynamic timing simulator and determined the signal delays at the observation points of the benchmarks. Next, we used MATLAB to compute the Kendall's correlation coefficients [32] for each pattern set. The Kendall's correlation coefficient is a measure between 0 and 1 , where 0 indicates no correlation and 1 indicates perfect correlation. Table $\mathrm{V}$ shows the average correlation coefficients for the patterns in a 1-detect test set of the IWLS'2005 benchmarks [8]. The results for other values of $n$ are similar. The minimum and maximum values of the correlation coefficients are also given. As seen in Table V, there is a strong positive correlation (close to the perfect correlation measure of 1) between output deviations and path lengths. Thus, the method of output deviations is a promising metric for evaluating the capability of transition delay-test patterns to sensitize long paths.

It can be argued that instead of output deviations, a dynamic timing simulator can be used to obtain high correlation to path lengths. However, the method based on output deviations
TABLE V

KENDALL'S COEFFICIENTS FOR EVALUATING THE CORRELATION OF PATH LENGThS to OUtPut DeViations

\begin{tabular}{|l|l||l|l|}
\hline Benchmark & $($ Ave, Min, Max) & Benchmark & (Ave, Min, Max) \\
\hline systemcaes & $(0.96,0.82,1)$ & pci_bridge32 & $(0.93,0.85,0.99)$ \\
\hline usb_funct & $(0.97,0.85,0.99)$ & wb_conmax & $(0.93,0.89,0.98)$ \\
\hline ac97_ctrl & $(0.98,0.93,1)$ & ethernet & $(0.89,0.77,0.95)$ \\
\hline aes_core & $(0.97,0.9,0.99)$ & & \\
\hline
\end{tabular}

is flexible and general, and it can be used during pattern selection to account for process variations and many physical defects. Dynamic timing simulation can only provide variability-unaware timing information. The method of output deviations is also expected to reveal unique problematic paths that may be hidden from dynamic timing analysis.

\section{G. Pattern Selection Results}

In this section, we present the pattern-selection results for the proposed deviation-based method (dev) and the dynamictiming simulation based method (dts) [15] for a production test environment with pattern-count limits.

We first generated 5-detect (n5), 8-detect (n8), and timingaware (ta) transition-test patterns for each benchmark using a 


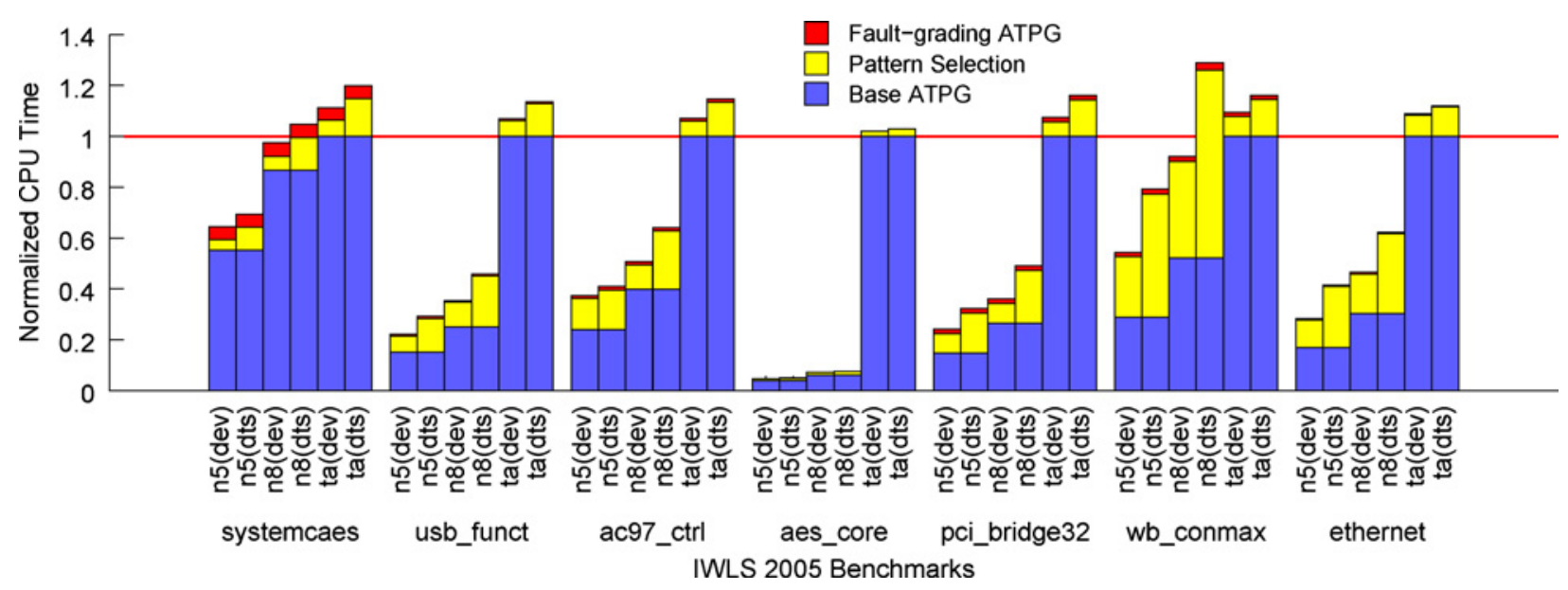

Fig. 6. Comparison of CPU time for dev and dts. The CPU times used for each step are shown in a different color. The values are normalized by the timing-aware ATPG CPU time for each benchmark. The normalization point (timing-aware ATPG CPU time) is shown as a red line.

TABLE VI

Number of Test Patterns Generated by the Commercial ATPG TOOL FOR VARIOUS $n$-DETECT TDF AND TIMING-AWARE ATPG (TA)

\begin{tabular}{|l|c|c|c|}
\hline Benchmarks & $\mathrm{n} 5$ & $\mathrm{n} 8$ & ta \\
\hline systemcaes & 1939 & 2821 & 2997 \\
\hline usb_funct & 3802 & 5797 & 3683 \\
\hline ac97_ctrl & 1907 & 2866 & 1643 \\
\hline aes_core & 2734 & 3995 & 8277 \\
\hline pci_bridge32 & 4789 & 7383 & 4571 \\
\hline wb_conmax & 21230 & 32189 & 6418 \\
\hline ethernet & 25221 & 36855 & 13544 \\
\hline
\end{tabular}

commercial ATPG tool. Next, dev and dts are used to select patterns from these base pattern sets. The motivation for also using timing-aware patterns as a base pattern-set lies in our observation that the pattern counts resulting from timing-aware ATPG for large industrial circuits are often prohibitively high. Test-set truncation is therefore necessary in practice. In order to obtain the same TDF coverage as 1-detect patterns, we ran top-off timing-unaware ATPG over the selected patterns.

The number of patterns generated by the commercial ATPG tool is given in Table VI. When dts is used as the pattern selection scheme, the top $1 / 3$ patterns (ranked on the basis of the "metric") of the base patterns (n5, n8, and ta) are selected as in [15]; see Table VII. The number of patterns after top-off ATPG for TDFs is given in parentheses.

For dev, we used a range of $D_{\text {LIMIT }}$ values for pattern selection. The results for $D_{\text {LIMIT }}=0.6$ and $D_{\text {LIMIT }}=0.8$ are shown in Table VIII. We see that an increase in $D_{\text {LIMIT }}$ resulted in a lower pattern count in the selected pattern set, but increased the number of top-off ATPG patterns. We observe that for most benchmarks, the selected pattern count is significantly smaller than the number of base timing-aware ATPG patterns, and even smaller than the number of patterns selected by dts. For the rest of the experimental results presented in this section for dev, we used the patterns selected with $D_{\text {LIMIT }}=0.8$.

The CPU time required by timing-aware ATPG is an important concern, especially for large industrial designs under time-to-market constraints. We have seen that for large
TABLE VII

Number of Test Patterns (ONE-ThiRd of BASE SeT) SELECTEd by THE DyNAMiC-Timing Simulation BASEd SCHEME (DTS)

\begin{tabular}{|l|c|c|c|}
\hline Benchmarks & $\mathrm{n} 5^{*}$ & $\mathrm{n} 8^{*}$ & $\mathrm{ta}^{*}$ \\
\hline systemcaes & $646(784)$ & $940(991)$ & $999(1151)$ \\
\hline usb_funct & $1267(1474)$ & $1932(2010)$ & $1226(1695)$ \\
\hline ac97_ctrl & $635(815)$ & $955(1019)$ & $551(783)$ \\
\hline aes_core & $911(1097)$ & $1331(1384)$ & $2759(2766)$ \\
\hline pci_bridge32 & $1596(1951)$ & $2461(2693)$ & $1507(2069)$ \\
\hline wb_conmax & $7076(7831)$ & $10729(10961)$ & $2142(5713)$ \\
\hline ethernet & $8407(10213)$ & $12284(12877)$ & $4514(9708)$ \\
\hline
\end{tabular}

*Numbers in parenthesis refer to the pattern count after top-off timingunaware ATPG.

industrial circuits, timing-unaware TDF ATPG itself can take a couple of days to complete. Therefore, the CPU time for timing-aware ATPG can be prohibitive, as shown in Fig. 1 for benchmark circuits. We evaluated the total CPU time used by dev and dts and compared it to the CPU time used by the base timing-aware ATPG. The results are shown in Fig. 6. We find that, even with CPU time for top-off ATPG, for $n$-detect pattern sets, dev consistently has lower CPU time compared to both base timing-aware ATPG and dts. For the ta pattern group, the CPU time used for pattern selection and fault-grading is significantly smaller than the base timing-aware ATPG run time.

In order to evaluate the effectiveness of selected patterns in terms of long-path sensitization, we ran timing simulations for the selected patterns and found the number of sensitized distinct long paths. Two paths are assumed to be distinct if there is at least one net that is not shared by them. A path is assumed to be a long path if the corresponding delay is higher than the specified long path limit (LPL). We ran the analysis for a range of LPL values, starting from $50 \%$ of the clock period to $90 \%$ of the clock period. The results for 90\% LPL is shown in Fig. 7. The results for other values of LPL follow the same trend. The contribution of the selected patterns and the top-off patterns is shown in different colors. Note that the number of patterns for the base timing-aware ATPG is much larger than any of the pattern selection methods. 
TABLE VIII

Number of Test Patterns Selected by the Deviation-Based Scheme (DeV) When $D_{\text {Limit }}=0.6$ and $D_{\text {Limit }}=0.8$

\begin{tabular}{|l|c|c|c|c|c|c|}
\hline & \multicolumn{3}{|c|}{$D_{\text {LIMIT }}=0.6^{*}$} & \multicolumn{3}{c|}{$D_{\text {LIMIT }}=0.8^{*}$} \\
\hline Benchmarks & $\mathrm{n} 5$ & $\mathrm{n} 8$ & $\mathrm{ta}$ & $\mathrm{n} 5$ & $\mathrm{n}$ & ta \\
\hline systemcaes & $974(1054)$ & $1135(1210)$ & $1245(1417)$ & $938(1027)$ & $1097(1174)$ & $1205(1374)$ \\
\hline usb_funct & $1171(1360)$ & $1330(1494)$ & $1298(1751)$ & $659(1110)$ & $742(1149)$ & $744(1390)$ \\
\hline ac97_ctrl & $614(740)$ & $699(805)$ & $575(801)$ & $281(589)$ & $246(582)$ & $63(544)$ \\
\hline aes_core & $1104(1214)$ & $1232(1309)$ & $1379(1479)$ & $1060(1182)$ & $1179(1277)$ & $1294(1413)$ \\
\hline pci_bridge32 & $1069(1525)$ & $1287(1683)$ & $1432(2005)$ & $408(1319)$ & $459(1341)$ & $504(1452)$ \\
\hline wb_conmax & $2434(5588)$ & $2572(5608)$ & $1949(5648)$ & $2091(5575)$ & $2275(5535)$ & $1525(5651)$ \\
\hline ethernet & $11967(13740)$ & $13448(14606)$ & $8877(11472)$ & $11479(13453)$ & $12911(14304)$ & $8493(11351)$ \\
\hline
\end{tabular}

*Numbers in parenthesis refer to the pattern count after top-off timing-unaware ATPG.

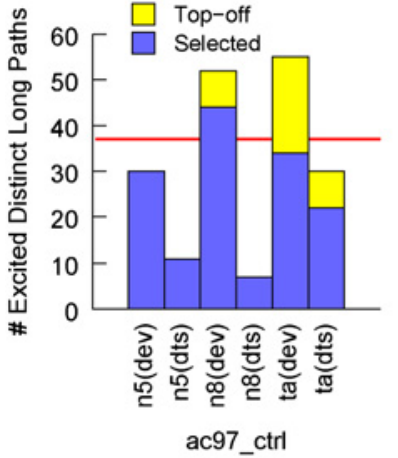

(a)

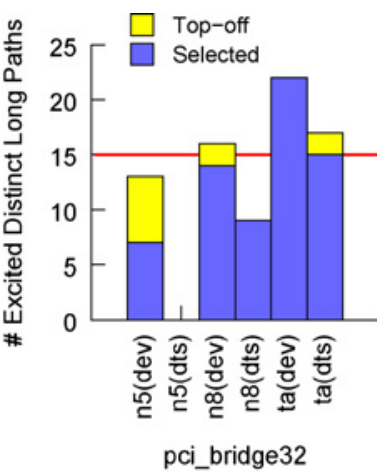

(b)

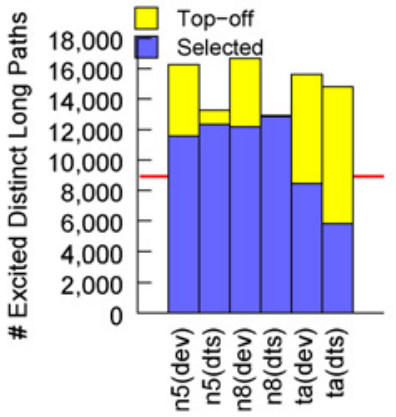

wb_conmax

(c)

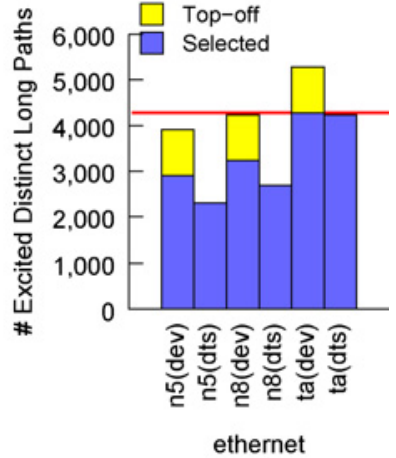

(d)

Fig. 7. Number of sensitized distinct long paths for dev and dts (LPL = 90\%). (a) ac97_ctrl (1643). (b) pci_bridge32 (4571). (c) wb_conmax (6418). (d) ethernet (13544). The number of sensitized distinct long paths for the trimmed timing-aware ATPG patterns is shown as a horizontal line. The number of sensitized distinct long paths for the full timing-aware ATPG pattern set is shown in parentheses next to benchmark names.

Therefore, we trimmed the base timing-aware ATPG patterns and selected the first $P$ patterns (ranked by the ATPG tool) as a baseline where $P$ is the maximum of the number of selected patterns (including top-off patterns) either by dev or dts. The results for the trimmed timing-aware ATPG patterns are shown as horizontal lines. The results for other benchmarks can be found in [9]. As seen in Fig. 7, dev consistently excites more long paths than dts. We also note that the proposed method sensitizes more long paths than the timing-aware ATPG baseline with $P$ patterns for three circuits-ac97_ctrl, pci_bridge32, and wb_conmax. It sensitizes nearly the same number of long paths for ethernet. Recall that in all cases, the CPU time for timing-aware ATPG is much higher (Fig. 6).

To further evaluate the long path sensitization capability of the selected patterns, we determined the delay distribution of the excited distinct long paths. We set LPL to $80 \%$ of the rated clock period. The results are shown in Fig. 8. The results shown in this figure are for the base 8-detect pattern set [n8 (base)], dts-based selected patterns and top-off patterns [n8 (dts)], and dev-based selected patterns with $D_{\text {LIMIT }}=0.8$ and top-off patterns $[\mathrm{n} 8(\mathrm{dev})]$. To draw appropriate conclusions, it is necessary to examine the pattern counts in Table VI (column n8), Table VII (column n8), and Table VIII (column n8 under $D_{\text {LIMIT }}=0.8$ ). Note that the base pattern set has a much larger pattern count compared to selected pattern sets with additional top-off patterns. Furthermore, dev-based selection leads to lower pattern count than dts for all benchmarks except systemcaes and ethernet. The difference in pattern count is especially significant for pci_bridge32 and wb_conmax, where dev-based selection has almost half of the pattern count of dts. We find that dev- based selection clearly outperforms dts-based selection for most benchmarks, even with fewer patterns. For wb_conmax, dev-based selection is almost as effective as dts-based selection even though the pattern count is only half. Another important observation is that dev-based selection successfully extracted almost all of the long path sensitizing patterns from the base pattern sets for most benchmarks. The results for other benchmarks can be found in [9].

To evaluate the effect of DDPM perturbations on the selected pattern quality, we ran perturbation analysis simulations. Fig. 9 shows the results for three representative benchmarks. For each benchmark, we injected random variations to DDPM entries using three different maximum variation values: $10 \%$ (p:0.1), 20\% (p:0.2), and 30\% (p:0.3). The injected variations are uniformly distributed between BASE- $p$ and BASE $+p$, where BASE shows the original DDPM entry (corresponding bar is named as p:0). The number of distinct long paths excited by the selected patterns is shown as a pattern quality metric. The number of excited distinct long paths for the corresponding full ATPG pattern sets (n5, n8, and timingaware) are shown as a red horizontal line. These lines simply show the maximum achievable limit for each selected pattern set (the blue bars under them). As seen in Fig. 9, the patternselection results and test quality are relatively insensitive to small changes in the DDPM entries. We attribute this finding to the fact that any DDPM changes affect multiple paths in the circuits, hence their impact is amortized over the circuit and the test set. It is usually very difficult to estimate close-toreal timing variations. The robustness of the deviation-based pattern selection against timing estimation errors can provide great flexibility in this aspect. 


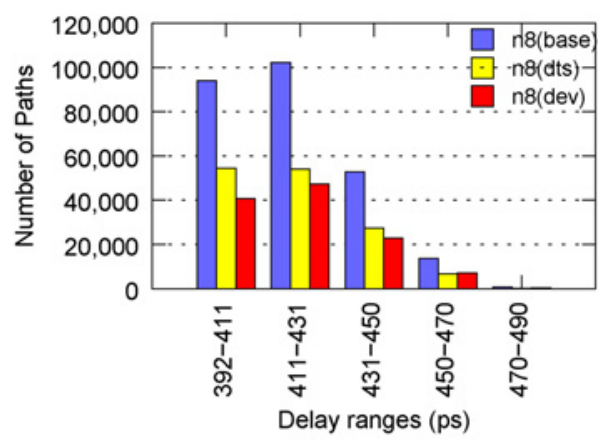

(a)

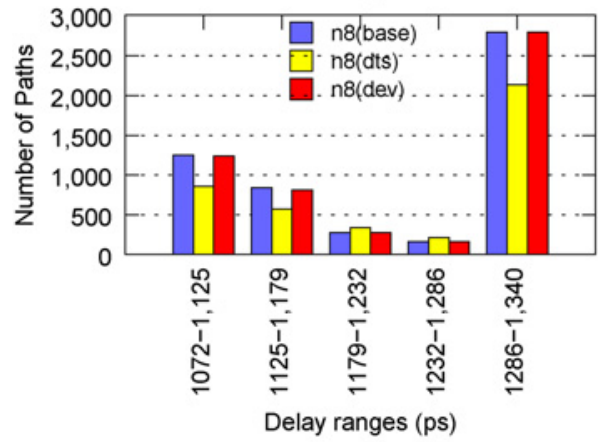

(b)

Fig. 8. Distribution of sensitized distinct paths (LPL $=80 \%)$ for base 8-detect patterns, dts-based selection, and dev-based selection. (a) wb conmax. (b) ethernet.

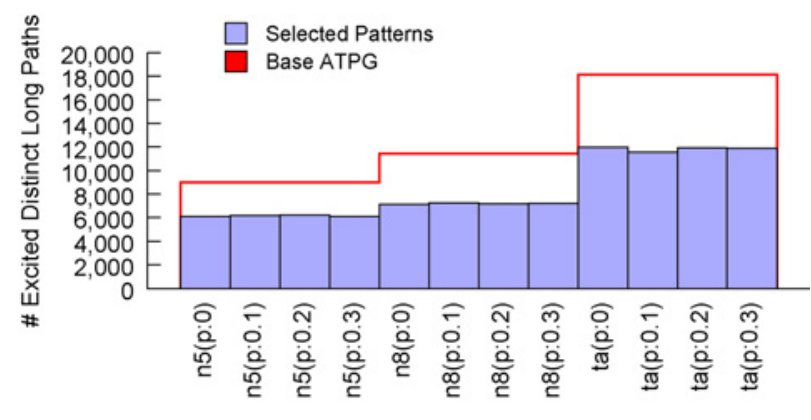

(a)

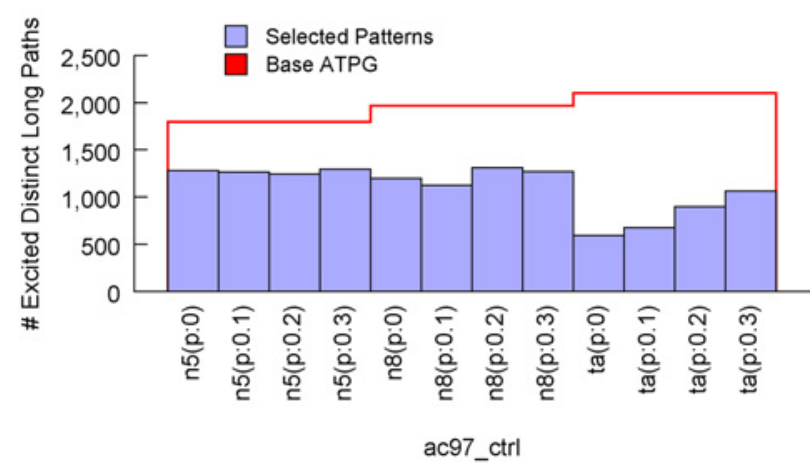

(b)

Fig. 9. Number of sensitized distinct long paths $(\mathrm{LPL}=70 \%)$ for selected patterns, under different DDPM perturbation limits: (a) usb_funct. (b) ac97_ctrl.

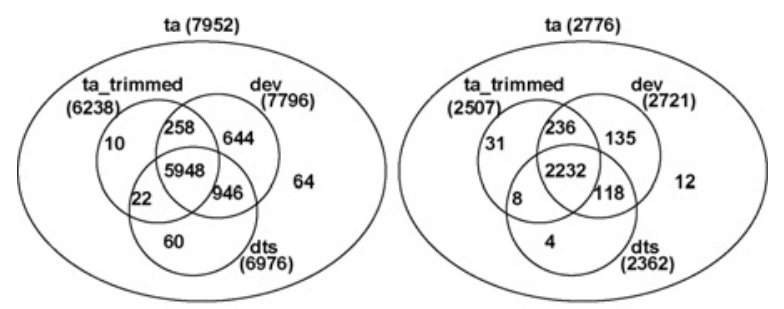

(a)

(b)

Fig. 10. Number of defects detected by the selected patterns for dev, dts, the trimmed timing-aware ATPG patterns (ta_trimmed), and full set of timing-aware ATPG patterns ( $\mathrm{ta}$ ) ( $\mathrm{ta}$ is a super-set of all selected patterns). (a) wb_conmax. (b) ethernet.

To evaluate the fault coverage ramp-up provided by dev, dts, and timing-aware ATPG, we ran fault injection simulations. For each benchmark, we inserted 50000 delay defects on randomly chosen nets. We assumed that the additional delay introduced by the injected defects has a distribution of $e^{-A x}$ as used in [10] and [29], and we injected random delay defects using the method described below.

We let $A=\frac{5}{T_{C L K}}$, where $T_{C L K}$ corresponds to the rated clock period of the circuit under test. The delay-defect distribution used in [10] and [29] is similar to this function. We use the distribution

$$
y=e^{\frac{-5 x}{T_{C L K}}} \quad 0<y<1 .
$$

If (5) is solved to determine $x$ (additional delay) in terms of $y$ (uniformly distributed random number), the following equation results:

$$
x=-\ln y \cdot \frac{T_{C L K}}{5} .
$$

A total of 50000 uniformly distributed random numbers were generated and corresponding delay defects were injected in the circuit under test. In our experiments, $70 \%$ of the injected delay defects are less than $20 \%$ of the clock period. Thus, our defect-injection mechanism injected more smalldelay defects than gross-delay defects, as is the case for VDSM technology [10].

The number of detected faults for all the benchmarks is presented as Venn diagrams in Fig. 10. Although it is only expected that dev patterns will not catch all detectable faults, we find that, for most cases, dev missed less faults compared to both dts and timing-aware ATPG.

Early detection of defects is also important in an aborton-first-fail methodology, and it can save considerable test time. Furthermore, if the test-time budget is limited, whereby truncation is necessary and only a small portion of the patterns can be used for production test, it is important to apply the most effective patterns before less effective ones. Fig. 11 shows how the fault coverage increases with the number of patterns for representative benchmark circuits. Each plot in these figures shows results for the base timing-aware ATPG patterns [ ta (base)], the patterns selected or sorted by dev [n8 (dev) and ta (dev)], and the pattern selected and sorted by dts [n8 (dts) and ta (dts)]. We find that the coverage rises more steeply for the proposed method (dev) compared to both dts and the base timing-aware ATPG patterns.

Finally, we have obtained results on long-path coverage and defect detection for the full timing-aware test sets, i.e., without truncation. The results are shown in [9]. As expected, the full 


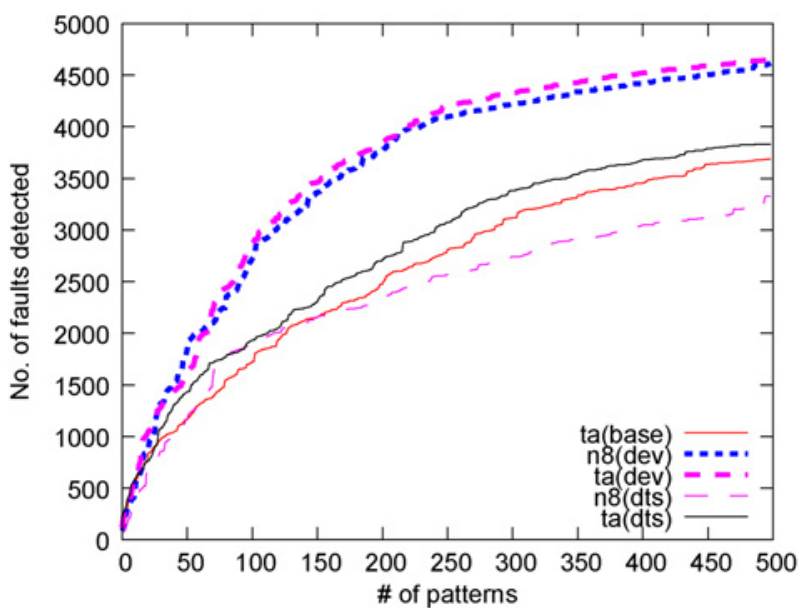

(a)

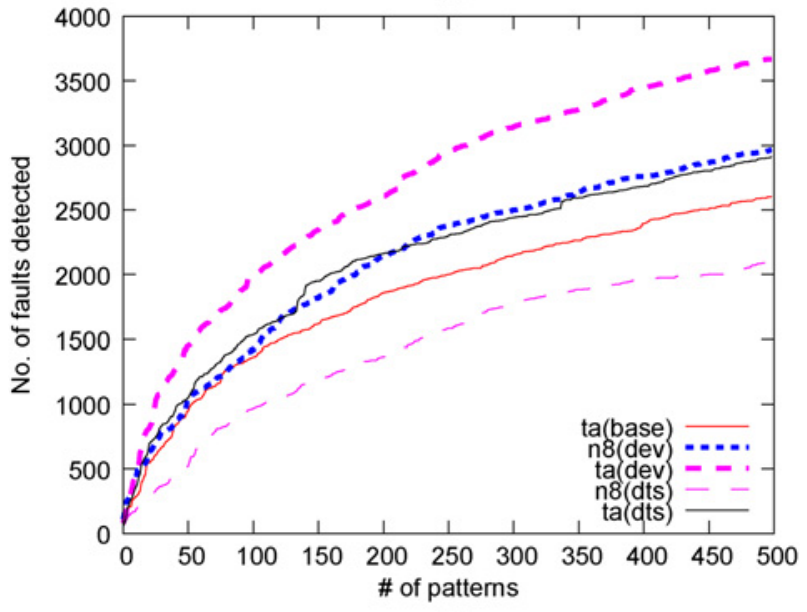

(b)

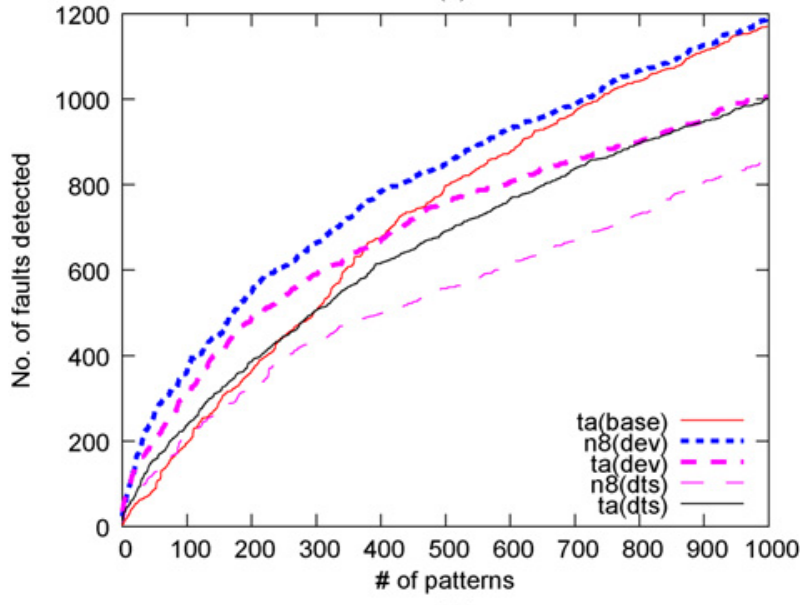

(c)

Fig. 11. Fault coverage ramp-up using the selected patterns of dev and dts, and the base timing-aware ATPG patterns. (a) usb_funct. (b) pci_bridge 32 . (c) ethernet.

timing-aware test sets provide higher coverage, but these test sets are much longer in length. For several benchmarks, comparable or even better coverage is achieved using the proposed method with much smaller test sets. For a range of smaller pattern counts, the proposed method clearly outperforms the full timing-aware test sets.

\section{CONCLUSION}

We have presented a test-grading technique, based on output deviations, for screening SDDs. We have defined the concept of output deviations for pattern-pairs and shown that it can be used as an efficient surrogate metric to model the effectiveness of TDF patterns for SDDs. We have introduced a gate-delay defect probability measure to model delay variations for nanometer technologies. Experimental results for the IWLS'2005 benchmark circuits show that the proposed method intelligently selects the best set of patterns for SDD detection from an $n$-detect or timing-aware TDF pattern set, and it excites a larger number of long paths compared to a current generation commercial timing-aware ATPG tool. We have also shown that the selected patterns are considerably more effective than a recently proposed method for detecting SDDs caused by resistive shorts, resistive opens, and process variations.

\section{ACKNOWLEDGMENT}

We would like to thank J. Rearick, J. Fitzgerald, and other colleagues at AMD for valuable discussions and for providing us with access to computing resources. We would also like to thank S. Patil from Intel for valuable discussions.

\section{REFERENCES}

[1] N. Ahmed, M. Tehranipoor, and V. Jayaram, "Timing-based delay test for screening small delay defects," in Proc. IEEE Design Autom. Conf., 2006, pp. $320-325$.

[2] X. Lin, K.-H. Tsai, C. Wang, M. Kassab, J. Rajski, T. Kobayashi, R. Klingenberg, Y. Sato, S. Hamada, and T. Aikyo, "Timing-aware ATPG for high quality at-speed testing of small delay defects," in Proc. IEEE Asian Test Symp., 2006, pp. 139-146.

[3] J. A. Waicukauski, E. Lindbloom, B. Rosen, and V. Iyengar, "Transition fault simulation," IEEE Design Test Comput., vol. 4, no. 2, pp. 32-38, Mar. 1987.

[4] R. Putman and R. Gawde, "Enhanced timing-based transition delay testing for small delay defects," in Proc. IEEE Very Large Scale Integr. Test Symp., 2006, pp. 336-342.

[5] Cadence, Inc. "Encounter test: Test generation and simulation reference," product Version 3.0, 2005.

[6] Mentor Graphics. "Understanding how to run timing-aware ATPG," Application Note, 2006.

[7] R. Kapur, J. Zejda, and T. W. Williams, "Fundamentals of timing information for test: How simple can we get?" in Proc. IEEE Int. Test Conf., Oct. 2007, pp. 1-7.

[8] IWLS 2005 Benchmarks [Online]. Available: http://iwls.org/iwls2005/ benchmarks.html

[9] M. Yilmaz, K. Chakrabarty, and M. Tehranipoor, "Test-pattern selection for screening small-delay defects in very-deep submicrometer integrated circuits,” Duke University, Tech. Rep. ECE-2009-02 [Online]. Available: http://hdl.handle.net/10161/1376

[10] B. Keller, M. Tegethoff, T. Bartenstein, and V. Chickermane, "An economic analysis and ROI model for nanometer test," in Proc. IEEE Int. Test Conf., 2004, pp. 518-524.

[11] F.-F. Ferhani and E. J. McCluskey, "Classifying bad chips and ordering test sets," in Proc. IEEE Int. Test Conf., Oct. 2006, pp. 1-10.

[12] Semiconductor Industry Association. (2007) International technology roadmap for semiconductors (ITRS) [Online]. Available: http:// www.itrs.net/links/2007itrs/home2007.htm

[13] Z. Wang and K. Chakrabarty, "Test-quality/cost optimization using outputdeviation-based reordering of test patterns," IEEE Trans. Comput.-Aided Design Integr. Circuits Syst., vol. 27, no. 2, pp. 352-365, Feb. 2008.

[14] Z. Wang and K. Chakrabarty, "An efficient test pattern selection method for improving defect coverage with reduced test data volume and test application time," in Proc. IEEE Asian Test Symp., 2006, pp. 333-338.

[15] H. Lee, S. Natarajan, S. Patil, and I. Pomeranz, "Selecting high-quality delay tests for manufacturing test and debug," in Proc. IEEE Int. Symp. Defect Fault Tolerance Very Large Scale Integr. Syst., 2006, pp. 59-70.

[16] E. S. Park, M. R. Mercer, and T. W. Williams, "Statistical delay fault coverage and defect level for delay faults," in Proc. IEEE Int. Test Conf., 1988, pp. 492-499. 
[17] P. Gupta and M. S. Hsiao, "ALAPTF: A new transition fault model and the ATPG algorithm," in Proc. IEEE Int. Test Conf., 2004, pp. 1053-1060.

[18] W. Qiu, J. Wang, D. M. H. Walker, D. Reddy, X. Lu, Z. Li, W. Shi, and H. Balachandran, "K longest paths per gate (KLPG) test generation for scan-based sequential circuits," in Proc. IEEE Int. Test Conf., 2004, pp. 223-231.

[19] S. Gurumurthy, R. Vemu, J. A. Abraham, and D. G. Saab, "Automatic generation of instructions to robustly test delay defects in processors," in Proc. IEEE Eur. Test Symp., 2007, pp. 173-178.

[20] H. Yan and A. D. Singh, "A new delay test based on delay defect detection within slack intervals (DDSI)," IEEE Trans. Very Large Scale Integr. (VLSI) Syst., vol. 14, no. 11, pp. 1216-1226, Nov. 2006.

[21] I. Pomeranz and S. M. Reddy, "Transition path delay faults: A new path delay fault model for small and large delay defects," IEEE Trans. Very Large Scale Integr. (VLSI) Syst., vol. 16, no. 1, pp. 98-107, Jan. 2008.

[22] S. Bose, H. Grimes, and V. D. Agrawal, "Delay fault simulation with bounded gate delay mode," in Proc. IEEE Int. Test Conf., Oct. 2007, pp. $1-10$.

[23] V. Iyengar, J. Xiong, S. Venkatesan, V. Zolotov, D. Lackey, P. Habitz, and C. Visweswariah, "Variation-aware performance verification using at-speed structural test and statistical timing," in Proc. IEEE Int. Conf. Comput.Aided Design, 2007, pp. 405-412.

[24] V. Zolotov, J. Xiong, H. Fatemi, and C. Visweswariah, "Statistical path selection for at-speed test," in Proc. IEEE Int. Conf. Comput.-Aided Design, 2008, pp. 624-631.

[25] C. Forzan and D. Pandini, "Why we need statistical static timing analysis," in Proc. IEEE Int. Conf. Comput. Design, 2007, pp. $91-96$.

[26] I. Nitta, S. Toshiyuki, and H. Katsumi, "Statistical static timing analysis technology," Fujitsu Sci. Tech. J., vol. 43, no. 4, pp. 516-523, Oct. 2007.

[27] C.-T. M. Chao, L.-C. Wang, and K.-T. Cheng, "Pattern selection for testing of deep submicron timing defects," in Proc. IEEE Design Autom. Test Eur., vol. 2. 2004, pp. 1060-1065.

[28] B. Lee, H. Li, L.-C. Wang, and M. Abadir, "Pattern selection for testing of deep submicron timing defects," in Proc. IEEE Int. Test Conf., vol. 2. 2005, pp. $1060-1065$.

[29] Y. Sato, S. Hamada, T. Maeda, A. Takatori, Y. Nozuyama, and S. Kajihara, "Invisible delay quality: SDQM model lights up what could not be seen," in Proc. IEEE Int. Test Conf., 2005, 9 p. 1210.

[30] M. Yilmaz, K. Chakrabarty, and M. Tehranipoor, "Interconnect-aware and layout-oriented test-pattern selection for small-delay defects," in Proc. IEEE Int. Test Conf., 2008, pp. 1-10.

[31] M. Yilmaz, "Automated test grading and pattern selection for small-delay defects," Ph.D. dissertation, Dept. Electr. Comput. Eng., Duke Univ., Durham, NC, Apr. 2009

[32] R. R. Wilcox, Fundamentals of Modern Statistical Methods: Substantially Improving Power and Accuracy, 2nd ed. New York: Springer, 2010, pp. $179-180$

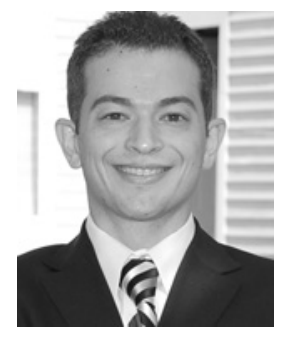

Mahmut Yilmaz (S'07-M'09) received the B.S degree in electrical and electronics engineering from Bogazici University, Istanbul, Turkey, in 2004, and the M.S. and Ph.D. degrees, both in electrical and computer engineering, from Duke University, Durham, NC, in 2006 and 2009, respectively.

Since 2006, he has been with Advanced Micro Devices, Inc., Sunnyvale, CA. He is currently a Senior Design Engineer with the Design-for-Test Team, Advanced Micro Devices, Inc. His current research interests include design for testability, physicalaware and timing-aware automatic-test-pattern generated for industrial digital circuits.

Dr. Yilmaz is the recipient of the Test Technology Technical Council Best Doctoral Thesis Award for 2009.

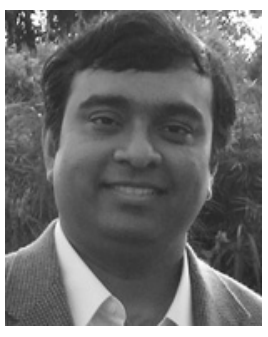

Krishnendu Chakrabarty (S'92-M'96-SM'02F'08) received the B.Tech. degree from the Indian Institute of Technology Kharagpur, Kharagpur, India, and the M.S.E. and Ph.D. degrees from the University of Michigan, Ann Arbor, in 1990, 1992, and 1995, respectively, all in computer science and engineering.

From 1995 to 1998, he was an Assistant Professor of electrical and computer engineering with Boston University, Boston, MA. He is currently a Professor of electrical and computer engineering with the Department of Electrical and Computer Engineering, Duke University, Durham, NC. He is a Member of the Chair Professor Group (honorary position) in
Software Theory with the School of Software, Tsinghua University, Beijing, China. He has authored nine books on these topics (including two in press), published over 320 papers in journals and refereed conference proceedings, and has given over 130 invited, keynote, and plenary talks. His current research interests include testing and design-for-testability of integrated circuits, digita microfluidics and biochips, circuits and systems based on deoxyribonucleic acid self-assembly, and wireless sensor networks.

Dr. Chakrabarty is a recipient of the National Science Foundation Early Faculty (CAREER) Award, the Office of Naval Research Young Investigator Award, the Humboldt Research Fellowship from the Alexander von Humboldt Foundation, Germany, and several best paper awards at the IEEE conferences. $\mathrm{He}$ is a Golden Core Member of the IEEE Computer Society, and a Distinguished Engineer of the Association for Computing Machinery (ACM). He is a 2009 Invitational Fellow of the Japan Society for the Promotion of Science. He is a recipient of the 2008 Duke University Graduate School Dean's Award for excellence in mentoring. He has served as a Distinguished Visitor of the IEEE Computer Society from 2005 to 2007, and as a Distinguished Lecturer of the IEEE Circuits and Systems Society from 2006 to 2007. Currently, he serves as an ACM Distinguished Speaker. He is an Associate Editor of the IEEE TRANSACTIONS ON COMPUTER-AIDED DESIGN OF INTEGRATED CIRCUITS AND SYSTEMS, the IEEE TRANSACTIONS ON VLSI SYSTEMS, and the IEEE TRANSACTIONS ON BIOMEDICAL CIRCUITS AND SYSTEMS. He also serves as an Editor of the Journal of Electronic Testing: Theory and Applications. He is the Editor-in-Chief of the IEEE DESIGN AND TEST OF COMPUTERS, and the ACM Journal on Emerging Technologies in Computing Systems.

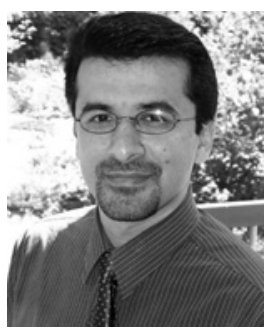

Mohammad Tehranipoor (S'02-M'04-SM'07) received the B.S. degree from the Amirkabir University of Technology (Tehran Polytechnic University), Tehran, Iran, the M.S. degree from the University of Tehran, Tehran, Iran, and the Ph.D. degree from the University of Texas at Dallas, Dallas, in 1997, 2000, and 2004, respectively, all in electrical engineering. $\mathrm{He}$ is currently an Assistant Professor of electrical and computer engineering with the Department of Electrical and Computer Engineering, University of Connecticut, Storrs. He has published over 85 journal articles and refereed conference papers in the area of very large scale integration (VLSI) design, test, and hardware security and trust. He has published two books entitled Nanometer Technology Designs High-Quality Delay Tests (Berlin, Germany: Springer) and Emerging Nanotechnologies Test, Defect Tolerance and Reliability (Berlin, Germany: Springer), in addition to three book chapters. His current research interests include computer-aided design and test for complementary metal-oxide-semiconductor VLSI designs, design-for-testability, at-speed test, secure design, and integrated circuit trust.

Dr. Tehranipoor is a recipient of the Best Paper Award at the 2005 VLSI Test Symposium (VTS), the Best Paper Award at the 2008 North Atlantic Test Workshop (NATW), the Best Paper Award at NATW in 2009, honorable mention for Best Paper Award at NATW in 2008, Best Paper candidate at the 2006 Design Automation Conference (DAC), Best Paper candidate at the 2005 Texas Instrument Symposium on Test, the Best Panel Award at VTS in 2006, and top ten paper recognition at the 2005 International Test Conference (ITC) $\mathrm{He}$ is also a recipient of the 2008 IEEE Computer Society Meritorious Service Award, the 2009 NSF CAREER Award, and the 2009 UConn ECE Research Excellence Award. He serves on the program committee of several leading conferences and workshops. He served as the Guest Editor for the Journal of Electronic Testing: Theory and Applications (JETTA) on "Test and Defect Tolerance for Nanoscale Devices" and the Guest Editor for the IEEE DESIGN AND TEST OF COMPUTERS on "IR-Drop and Power Supply Noise Effects on Very Deep-Submicrometer Designs." He served as the Program Chair of the 2007 IEEE Defect-Based Testing Workshop, the Program Chair of the 2008 IEEE Defect and Data Driven Testing (D3T), the Co-program Chair of the 2008 International Defect and Fault Tolerance in VLSI Systems (DFT), and the General Chair for D3T in 2009 and DFT in 2009. He co-founded a new workshop called the IEEE International Workshop on Hardware-Oriented Security and Trust (HOST) and served as the HOST-2008 and HOST-2009 General Chair and Chair of Steering Committee. He is currently an Associate Editor for the JETTA, an Associate Editor for the IEEE DESIGN AND TEST OF COMPUTERS, and an Editor for the TTTC NEWSLETTER. He is a Member of Association for Computing Machinery (ACM) and the ACM Special Interest Group on Design Automation. 